\title{
RECIDIVA DA HÉRNIA INCISIONAL APÓS O TRATAMENTO PELA TRANSPOSIÇÃO PERITÔNIO-APONEURÓTICA LONGITUDINAL BILATERAL
}

\author{
Alcino LÁZARO-da-SILVA, Rodrigo Garcia VIEIRA e Gustavo Coelho dos ANJOS
}

RESUMO - Objetivo - Conhecer a incidência de recidiva de hernioplastia incisional pela transposição peritônio-aponeurótica longitudinal bilateral. Procedimentos - Foi feito um levantamento dos pacientes que foram operados de hérnia incisional pela técnica de transposição peritônio-aponeurótica longitudinal bilateral. Cento e trinta e dois pacientes ( 80 do Hospital Universitário de Belo Horizonte, MG e 52 da clínica particular) foram acompanhados por período médio de 4 anos e 10 meses. Muitos dos pacientes eram obesos, porém todos tinham hérnias longitudinais de médio a grande volume. Três aspectos precisam ser ressaltados: o método para se alcançar a parede abdominal interna, a incisão aponeurótica posterior e os fios utilizados. Resultados - Recidiva em 7,69\% dos pacientes do grupo operado por um cirurgião (clínica particular) e em 18,75\% dos pacientes do grupo operado por cirurgiões em treinamento (Hospital Universitário).

DESCRITORES - Hérnia ventral, cirurgia. Recidiva.

\section{INTRODUÇÃO}

Em 1971 comunicamos uma proposição técnica para o tratamento de hérnia incisional $(\mathrm{HI})^{(13,16)}$. Trata-se de uma transposição peritônio-aponeurótica longitudinal bilateral (Transpalb). A experiência aumentou, reforçada por investigações

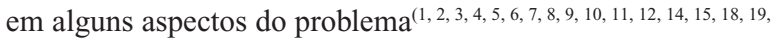
$20,21,22,23,24,25,26,27,28)$.

Os resultados tardios foram positivos, propiciando a continuidade do seu uso ${ }^{(23)}$. Com isso, passados alguns anos, avaliamos no pós-operatório tardio, a incidência de recidivas, que é o objetivo deste trabalho.

\section{PACIENTES E MÉTODOS}

Entre a experiência adquirida, analisamos os prontuários de 132 pacientes (31 homens e 101 mulheres) operados pela Transpalb, que foram acompanhados no pós-operatório tardio (Tabela 1).

Todas as hérnias eram longitudinais e apresentavam volumes
TABELA 1 - Epidemiologia da hérnia incisional

\begin{tabular}{lcc}
\hline Sexo & Masculino & $31(23,5 \%))$ \\
& Feminino & $101(76,5 \%)$ \\
\hline Etnia & Melanodérmico & $20(15,2 \%)$ \\
& Feodérmico & $53(40,2 \%)$ \\
& Leucodérmico & $59(44,6 \%)$ \\
\hline Idade* & de 20 a 39 anos & $14(10,8 \%)$ \\
& de 40 a 59 anos & $71(54,7 \%)$ \\
& de 60 a 79 anos & $43(33,0 \%)$ \\
& 80 ou mais & $2(01,5 \%)$ \\
\hline Estado civil & Casado & $92(69,7 \%)$ \\
& Solteiro & $17(12,9 \%)$ \\
& Outros & $23(17,4 \%)$ \\
\hline
\end{tabular}

* 1 paciente com idade de 3 anos e 1 outro sem relato de idade

médios e grandes, predominando em pacientes obesos e com perda de domicílio.

O tempo de controle variou de 6 meses a 22 anos, com a média de 4 anos e 10 meses.

Departamento de Cirurgia da Universidade Federal de Minas Gerais, Belo Horizonte, MG

Endereço para correspondência; Dr. Alcino Lázaro da Silva - Rua Guaratinga, 151 - Sion - 30315-430 - Belo Horizonte, MG. 
A localização anatômica preferencial das hérnias incisionais está indicada na Tabela 2.

TABELA 2 - Localização da hérnia.

\begin{tabular}{lccc}
\hline Localização & Paramediana & Mediana & n (\%) \\
\hline Supra-umbilical & $7(5,3 \%)$ & $27(20,5 \%)$ & $34(25,8 \%)$ \\
Infra-umbilical & $10(7,6 \%)$ & $55(41,6 \%)$ & $65(49,2 \%)$ \\
Supra e infra-umbilical & $2(1,5 \%)$ & $7(05,3 \%)$ & $9(06,8 \%)$ \\
Supra-médio-infra-umbilical & - & $3(02,3 \%)$ & $3(02,3 \%)$ \\
Médio-umbilical & - & $9(06,8 \%)$ & $9(06,8 \%)$ \\
Sem relato de localização & - & - & $12(9,10 \%)$ \\
Total de casos & $19(14,4 \%)$ & $101(76,5 \%)$ & $132(100,0 \%)$ \\
\hline
\end{tabular}

Os tempos operatórios foram padronizados e descritos em publicações anteriores $^{(5,7,15)}$.

Houve três aspectos que devem ser ressaltados: o acesso na pele foi feito por incisão longitudinal ou transversal, quando associada à dermolipectomia; a incisão aponeurótica posterior, na maioria das vezes, foi feita atrás do músculo reto do abdome à esquerda, e os fios, que variaram na sua qualidade, preferencialmente foram os absorvíveis.

No pré e pós-operatório, os pacientes foram tratados como os que se submetem a intervenções de porte médio.

Em geral, usa-se faixa de crepom ou cinta elástica de média compressão, para dar mais conforto e facilitar a movimentação, até o segundo mês. O levantar do leito foi precoce, ocorrendo freqüentemente após o segundo dia pós-operatório.

Não se usou pneumoperitônio nos pacientes constantes nesta casuística.

As coleções subcutâneas ou supurações foram aspiradas por punção ou drenadas por contra-abertura. As raras epidermólises foram desbridadas ou tratadas conservadoramente para granulação e epitelização natural. $\mathrm{Na}$ revisão tardia, o exame do abdome foi feito nas condições normais de uma propedêutica e, em pé, à procura de nódulos ou anéis.

\section{RESULTADOS}

As intercorrências pós-operatórias imediatas estão listadas na Tabela 3.

Observa-se na Tabela 4 que há uma diferença nítida entre a casuística de um só cirurgião ( 52 pacientes e 4 recidivas, 7,69\%), e a de vários outros em período de formação técnica ( 80 pacientes e 15 recidivas, $18,75 \%$ ). O último exame clínico revelou os resultados constantes na Tabela 4. Houve uma taxa de recidiva de $13,22 \%$ dos casos operados pela Transpalb.

TABELA 3 - Pós-operatório imediato da Transpalb

\begin{tabular}{lc}
\hline Sem intercorrências & $105(79,55 \%)$ \\
Infecção de F.O. & $6(04,55 \%)$ \\
Deiscência de sutura & $3(02,30 \%)$ \\
Diástase de M.R.A. & $5(03,80 \%)$ \\
Íleo funcional & $1(0,75 \%)$ \\
Seroma & $6(4,50 \%)$ \\
Hematoma & $1(0,75 \%)$ \\
Insuficiência renal aguda (óbito) & $1(0,75 \%)$ \\
Flacidez de fossa inguinal d. e e. & $1(0,75 \%)$ \\
Peritonite & $3(2,30 \%)$ \\
\hline
\end{tabular}

Todos estes pacientes apresentaram hérnias recidivadas pequenas e nos ângulos da cicatriz, bem menores do que as originais e oligossintomáticas. Na maioria dos casos, ocorreram no ângulo inferior da área operada (Tabela 4).

TABELA 4 - Localização da recidiva

\begin{tabular}{lccc}
\hline & Hospital Universitário & Clínica particular & Total \\
\cline { 2 - 4 } Ângulo superior & $4(21,0 \%)$ & $0(00,0 \%)$ & $4(21,0 \%)$ \\
Ângulo inferior & $7(37,0 \%)$ & $4(21,0 \%)$ & $11(58,0 \%)$ \\
$1 / 3$ médio & $4(21,0 \%)$ & $0(00,0 \%)$ & $4(21,0 \%)$ \\
Total & $15(79,0 \%)$ & $4(21,0 \%)$ & $19(100,0 \%)$ \\
\hline
\end{tabular}

\section{DISCUSSÃO}

A solução anatomofuncional e definitiva para a hérnia incisional, ainda está por vir. A preocupação dos cirurgiões é a de obter o seu controle e diminuir o índice de recidiva, porque evitá-la por completo ainda não se conseguiu.

Nos dias atuais, a literatura registra incidência de 7,4\% a 13,2\% de hérnias incisionais para feridas limpas, e $15 \%$ a $31 \%$, para feridas contaminadas ${ }^{(10)}$. Nos relatos apresentados, a taxa de recidiva das $\mathrm{HI}$ operadas oscilou de $5 \%$ a $44 \%$ dos casos, com os mais variados métodos operatórios ${ }^{(11)}$.

O índice que encontramos foi de 13,22\% em período de 4 anos e 10 meses, em média. Comparando-se os nossos resultados com a literatura podemos estar certos de que a técnica em estudo tem o menor índice de recidiva, quando se consideram os seguintes aspectos:

- treinamento do cirurgião e sua experiência com determinada técnica tem muita importância nos resultados tardios, conforme a Tabela 4 pode demonstrar;

- pacientes obesos na segunda metade da vida;

- hérnias com volumes grandes ou médios e perda de domicílio, na maioria;

- não se usou artifício pré-operatório como, por exemplo, o pneumoperitônio;

- não se usou prótese e nem enxerto de tecido autólogo;

- não houve necessidade de usar fios especiais;

- não houve complicações graves, exceto um óbito por insuficiência renal aguda;

- não houve pacientes com insuficiência respiratória ou que exigissem cuidados clínicos intensivos;

- pode-se associar operações intra-abdominais ou dermolipectomia sem comprometimento da segurança;

- as recidivas foram de tamanho pequeno e sempre nos ângulos, especialmente no inferior;

- uma delas foi corrigida com pequena lâmina de prótese sintética que não controlou nova recidiva;

- nas reoperações para a cavidade abdominal, a parede mostra-se firme e os músculos retos do abdome ficam bem recobertos pelas neobainhas;

- acima ou abaixo do anel da recidiva, o tecido é firme e rígido, demonstrando que houve bom reforço cicatricial, e as falhas foram devidas à execução técnica ou a locais menos guarnecidos por tecidos mais resistentes.

Julgamos que a técnica, apesar de algumas recidivas, oferece uma excelente alternativa aos cirurgiões. É facilmente exeqüível, sem auxílios tecnológicos, em qualquer ambiente modesto mas responsável e por cirurgiões de experiência mediana. 
Lázaro-da-Silva A, Vieira RG, Anjos GC. Recidivation of bilateral longitudinal peritoneum-aponeurotic transposition on incisional hernioplasty. Arq Gastroenterol 2004;41(2):134-6.

ABSTRACT - Objectives - To carry out a study of the recidivation rate of incisional hernioplasty by means of bilateral longitudinal peritoneum-aponeurotic transposition. Method - A retrospective study was carried out in patients from the University Hospital in Belo Horizonte, MG, Brazil and from a surgeon's private clinic who had undergone incisional hernioplasty by means of bilateral peritoneum-aponeurotic transposition. A total of 132 patients $(80$ from University Hospital and 52 from private clinic) were monitored over an average period of 4 years and 10 months. Most the patients were obese and all of them had disloged longitudinal hernias of medium and large volume. Three aspects must be stressed: the procedure for reaching the internal abdominal wall, the posterior aponeurotic incision and the threads used. Results - Recidivation occurred in $7.69 \%$ of the patients belonging to a group operated on by one surgeon and in $18.75 \%$ of patients operated on by a trainee surgeon.

HEADINGS - Hernia, ventral, surgery. Recurrence.

\section{REFERÊNCIAS BIBLIOGRÁFICAS}

1. Barroetaveña J, Herszage L - Cirugía de las eventraciones. Buenos Aires, Argentina: El Ateneu; 1988. p.121-4

2. Browse NL, Hust P. Repair of long large midline incisional hernias using reflected flaps of anterior rectus sheath reinforced with marlex mesh. Am J Surg 1979;138:738.

3. Camargo SJ. Cirurgia ginecológica. Porto Alegre: Fundo Editorial Byk; 1991. p.82-3.

4. Cataldo MLS. Emprego do saco herniário na correção cirúrgica das hérnias incisionais longitudinais. Aspectos experimentais [dissertação]. Belo Horizonte: Faculdade de Medicina da Universidade Federal de Minas Gerais; 1977.

5. Cataldo MLS, Lázaro-da-Silva A, Guerra AJ. Emprego de saco herniário na correção cirúrgica das hérnias incisionais longitudinais. Aspectos experimentais. Rev Col Bras Cir 1981;8:167-70

6. Cougard P, Douvier S, Thomas T, Ferry C. Traitment des éventrations médianes Technique de Lázaro da Silva. Lyon Chirurgical 1986;82:66-8.

7. Escalante JR, Diogo-Filho A, Andrade JI, Faria LP, Pacheco RC, Nascimento RS, Correa M. Tratamento das hérnias incisionais volumosas pela técnica de Lázaro da Silva. Rev Col Bras Cir 1983;10:24-8.

8. Faria LP, Lázaro-da-Silva A. Hérnias incisionais medianas e paramedianas; estudo do saco herniário a microscopia óptica (mesotélio, tecido conjuntivo frouxo e denso com presença de fibras colágenas, reticulares e elásticas). Rev Col Bras Cir 1996;73:187-91.

9. Hope PG, Carter SS, Kilby JO. The da Silva method of incisional hernia repair. Br J Surg 1985;72:569-70.

10. Kather-Neto JM, Lázaro-da-Silva A. Cálculo de área e volume do saco herniário da hérnia incisional longitudinal - aplicação no planejamento cirúrgico. Rev Bras Cir 1987;77:213-8

11. Katter-Neto JM. Análise de 90 pacientes portadores de hérnia incisional operados pela técnica de Lázaro da Silva. Rev Col Bras Cir 1993;20:251-5.

12. Langer S, Christiansen J. Long-term results after incisional hernia repair. Acta Chir Scand 1985;151:217-9.

13. Lázaro-da-Silva A. Plástica com o saco herniário na correção cirúrgica das hérnias incisionais. O Hospital 1971;79:123-34.

14. Lázaro-da-Silva A. Plástica com o saco herniário na correção das hérnias incisionais longitudinais medianas ou pararretais e nas diástases dos retos abdominais. Rev Col Bras Cir 1974;3:113-6.
15. Lázaro-da-Silva A. Comentário sobre a superposição peritônio-aponeurótica bilateral com o saco herniário das hérnias incisionais longitudinais medianas e paramedianas. Rev Assoc Med Bras 1979;25:87-90.

16. Lázaro-da-Silva A. Surgical correction of longitudinal median or paramedian incisional hernia. Surg Gynecol Obstet 1979;148:579-83.

17. Lázaro-da-Silva A, Tobón MJC, Lima MJV, Ferreira AP. Evaluación radiológica de la cirugía de la hernia incisional. Trib Med (Bogotá) 1985;33-5.

18. Lázaro-da-Silva A. Bilateral surgical correction of longitudinal median, paramedian internal and external pararectal incisional hernias and diastasis. ABCD Arq Bras Cir Dig 1987;2:60-3

19. Lázaro-da-Silva A, Ferreira AP, Ribeiro V. Estudo eletromanométrico em pacientes portadores de hérnia incisional. Rev Col Bras Cir 1990;17:2-4.

20. Lázaro-da-Silva A. Incisional hernia [letter]. J Pediatr Surg 1991;26:1261.

21. Lázaro-da-Silva A, Lanna JCBD, Tatsuo ES, Guimarães JT. Emprego do saco herniário na correção cirúrgica da hérnia incisional longitudianal na criança (uma observação). Folha Med 1991;103:73-6.

22. Lázaro-da-Silva A, Petroianu A. Incisional hernias: factors influencing development. South Med J 1991;84:1500, 1504

23. Lucarotti M, Billings P, Leaper DJ. Laparotomy, wound closure and repair of incisional hernias. The da Silva repair. In: General surgery. The Medicine Group Journal; 1992. v.16, p.1-6

24. Morais AAC, Paulo DNS, Guerra AJ, Gama LPN, Laurentino PR. Técnica da interposição peritônio-aponeurótica bilateral no tratamento das grandes hérnias incisionais. Rev Bras Cir 1985;75:219-23.

25. Paulo DNS, Lázaro-da-Silva A. Repercussões respiratórias funcionais da hernioplastia incisional abdominal longitudinal. Rev Col Bras Cir 1995;22:33-41.

26. Paulo DNS, Lázaro-da-Silva A, Paulo ICAL, Rosolem F. Estudo da função respiratória em pacientes obesos e não obesos. Rev Bras Cir 1995;89:89-128.

27. Pitrez FAB, Lemchen HF, Furtado JP. Reconstrução da parede abdominal em eventrações. In: Bonamigo TP, editor. Condutas cirúrgicas. Porto Alegre: Fundação Faculdade Federal de Ciências Médicas de Porto Alegre; 1993. p.151-4.

28. Vidal Sans J. Eventraciones. Procedimentos de reconstrucción de la pared abdominal. Barcelona: JIMS; 1986. p.75.

Recebido em 25/4/2003. Aprovado em 1/8/2003. 